\title{
Performance Study of Locality and Its Impact on Peer-to-Peer Systems
}

\author{
Mohammed Younus Talha, Rabah W. Aldhaheri, Mohammad H. Awedh \\ Department of Electrical and Computer Engineering, King Abdulaziz University, Jeddah, Saudi Arabia \\ Email: ymohammed0003@stu.kau.edu.sa, raldhaheri@kau.edu.sa, mhawedh@kau.edu.sa
}

Received 15 January 2015; accepted 30 January 2015; published 2 February 2015

Copyright (C) 2015 by authors and Scientific Research Publishing Inc.

This work is licensed under the Creative Commons Attribution International License (CC BY). http://creativecommons.org/licenses/by/4.0/

c) (i) Open Access

\begin{abstract}
This paper presents the measurement study of locality-aware Peer-to-Peer solutions on Internet Autonomous System (AS) topology by reducing AS hop count and increase nearby source nodes in P2P applications. We evaluate the performance of topology-aware BT system called TopBT with BitTorrent (BT) by constructing AS graph and measure the hops between nodes to observe the impact of quality of service in P2P applications.
\end{abstract}

\section{Keywords}

Peer-to-Peer, BitTorrent, TopBT, Locality, Autonomous System

\section{Introduction}

Peer-to-Peer (P2P) is a distributed computing model which aims to share resources whose concept is not completely new. However, P2P systems are natural evolution in decentralized system architecture [1] in which peer is a node that can act as a client and server simultaneously in dynamic environment. Nodes can join or leave the system freely and also exchange resources directly without the help of a third party server. Popular P2P systems generate massive amount of traffic over the internet and it has been reported that $65 \%-70 \%$ Internet backbone is P2P traffic. Furthermore, it may be estimated that 50\% - 65\% of download traffic and $75 \%-90 \%$ of upload traffic is generated by P2P traffic access communities [2]. P2P networks can be classified according to their functionalities into three main classes such as file sharing, video streaming and VoIP. File sharing P2P applications like BitTorrent (BT), TopBT are the most popular among the three classes whereas video streaming classes applications are PPLive, PPStream and Voip application is Skype.

Zatto [3] was introduced as a localized P2P live streaming system and Skype [4] was modified to implement locality in super peers selections. Finally, Top-BT [5] was introduced as localized version of the BitTorrent software which is developed by OHIO State University R\&D Dept., that actively discovers its network proximi- 
ties to its connected peers, this unique feature separates it from Bit torrent.

It also improves the peers transmission rate of network for a faster download, reduces topology un-awareness due to unnecessary traffic and maintains faster download speed compared to other clients [6]. BitTorrent which is a well-known non-localized file sharing software in P2P networking used in common for transferring large file in a vast community environment with an exceptional download speed [7].

The popularity of P2P applications had massive traffic load that revealed doubts about the ability of internet service provider (ISP) that carried P2P traffic [8] and to sustain their cost of transit traffic. Due to these reasons and others inspired research to replace P2P random algorithms with locality-awareness algorithms where locality is a distance measurement method that can be utilized to express locality awareness.

Peer-to-peer (P2P) locality has recently raised lots of interest locally as its written content distribution dramatically raises the traffic within the inter-ISP links, in order to solve this problem the idea to keep a fraction in the P2P site visitors local to help each ISP has been introduced a couple of years ago. Several fundamental issues on locality are being explored such as measuring the content distribution and knowing the harmful effect of locality which intensify the demand of the content file that is shared on the network.P2P applications and ISPs have different lanes of business models that attempt to attract more users by increasing quality of service (QoS).

The fact that allowed P2P application developers to consider underlying networks as free resources and on the other hand ISP's attempting to drag down their inter/intra-domain traffic to increase their profits [9]. This business model authorized ISP's consider P2P applications as a harmful services and thus started to domesticate them by blocking their traffic with the help of shaping devices [5] and on the other side P2P applications counter-strike by encrypting their traffic using port hoping that leads to endless chasing.

However to tackle ISP issues, Autonomous system (AS) hops can be utilized to harvest AS-level to pology information and closely relate the AS-based ISP pricing model. Locality awareness algorithm implementation in P2P application was widely studied in the past years [10]-[12]. Each network on the Internet is recognized by a unique identifier known as Autonomous system number (ASN) which owns a set or a block of Internet Protocol (IP) addresses that have been assigned to it, in order to prevent traffic from propagation, content should be exchanged with other IP addresses in the same AS.

Sniffing is one of the most effective techniques in attacking a wireless network. Sniffer [13] is a program that eavesdrops on the network traffic by grabbing information that travels over a network and the Source for many network-based attacks is passive sniffing. Passive sniffing involves employing a sniffer to be able to monitor these kinds of incoming packets which uses a feature connected with network greeting cards called promiscuous mode. In this mode a network card will pass all packets on the operating structure, rather than those Unicast as well as broadcast towards host [13].

World's prime network protocol analyzer named Wireshark [14] enables to capture and interactively browse the traffic flowing on a computer network. This software is customary across many industries and educational institutions. Wireshark uses a packet capture in short Pcap, an application programming language to capture packets, so it can only capture the packets on the types of networks that Pcap supports. Yi Cui, et al., [15] proposes locality awareness in bit torrent like P2P applications which proposes an optimal solution with minimum AS hop count distribution structure and also describes that seeding cannot improve standard bit torrent download time but can improve its locality policies significantly.

The paper is organized as follows. Section 2 depicts the methodology of achieving goals of our study. Results analysis and performance of Locality and TopBT is studied and discussed in Section 3. Finally, Section 4 gives conclusion and possible future work to improve the quality of service in P2P applications.

\section{Methodology and Data Collection}

Our methodology of collecting data is to download Torrent files using two different file sharing applications such as Bit Torrent and Top-BT, which were operated in two separate computers. The download time of both Torrent clients was calculated and recorded simultaneously. Wireshark captures and save data packets of both Torrent clients. Then, a utility software was used to extract source and destination IP addresses form Wireshark captured files.

AWK tool [16] was used to delete the duplicate of the source and destination IP addresses. Cymru tool [17] had been utilized by which IP addresses were converted into Autonomous System Numbers (ASN). Java code was developed to find the AS paths from source IP address to destination IP address. By extracting these paths, 
we then compared the paths generated by the BitTorrent and Top-BT applications. This procedure had been applied on different file formats (Audio, Video, Application files, etc.). Hence the whole data was collected at particular geographical location.

During the downloading of some files we came across a huge download time duration by which a user may lose interest in downloading that particular file. Investigation of the scenario was done to show the impact of locality on the quality of experience. Our calculation was based on the Autonomous System Number and after gathering these numbers, AS paths has been our metric to measure locality.

The following steps were taken to achieve our goal. First we reviewed the concepts of Peer to Peer network (P2P) and Locality revision on the P2P applications.Then, Wireshark and AWK software tools were used for data collection. Finally, Java program and Cymru software tool were employed for data analysis part by which IP addresses were extracted and has been converted to Autonomous System Numbers (ASN).

Two well-known P2P file sharing systems were utilized, namely, Bit Torrent and Top-BT. Software programming and simulation tools such as Java, AWK, Cymru were adapted to map IP's into AS numbers. MSExcel has been used to show AS paths as final output by which the paths between Bit-torrent and TopBT are compared to measure their QoS and Locality in a P2P network.

\section{Results}

Locality awareness has emerged as the anchor to tackle the unwanted traffic issue where locality awareness algorithms allow peers to measure their distances from other nodes and utilizes this knowledge in selecting near content sources. To implement this algorithm, many issues must be tackled. For example, how to measure distances? How to find location? How to define near nodes and far nodes?

Collecting underlying network measurements and utilizing this information is the way to answer the previous questions. Peer should have the ability to measure the AS hop count path to reach different peers and must have the ability to map IP addresses into their AS numbers that can be able to measure delay, bandwidth and loss in the path. Finally, they should have algorithms that utilize this information (Locality algorithm). The main objective of locality awareness studies is to construct a P2P system that satisfies the requirement of ISPs by reducing the hops count and increase the number of local source nodes in one way and on the other end there shouldn't be impact on quality of experience in P2P networks.

Our results show that the average AS hops count path between neighbors in TopBT platform is shorter than the distances between neighbors in BT network. In addition, we have observed from our results that locality awareness implemented in TopBT has impact in reducing the intra-domain traffic that passes between AS's. Unfortunately, the implementation of locality awareness algorithm may reduce the performance, Quality of Service (QoS) and Quality of Experience (QoE), of P2P networks if the required content is unpopular.

In other words, the popularity of file in P2P file sharing network may affect implementation of locality awareness algorithm which means that the locality awareness algorithm requires a popularity of files to increase the performance of P2P applications or it will decrease its normal performance. In our measurement study we have obtained our results for Autonomous System paths of sources and destinations on Inter Autonomous System level routing.

In Figure 1 we have evaluated average Autonomous System paths of Audio files in which TopBT has better download rate than BitTorrent. Figure 2 and Figure 3 shows the AS source path comparison of Video files and Application files respectively whereas the Video files that contain large data size results in such case TopBT has performed good as shown in Figure 2. Finally, we note that Figure 4 show Document source average AS path files.

In P2P networks, nodes act as client and server simultaneously. Figure 5 shows average AS paths of Audio destination files, in Figure 6 and Figure 7 average AS path destination files of Video and Application are shown in which the performance of BitTorrent is slight better than TopBT. However TopBT had good performance overall, whereas Figure 8 shows the average AS hops path of destinations of Document files to compare the performance of TopBT with BitTorrent.

By observing these average AS paths of source files and destinationfiles figures respectively we can list out our findings, At first we noticed average AS hops paths of TopBT is shorter in most of the cases. However, in some cases this path is longer than BitTorrent. The reason is that the downloaded files in these cases are not popular, which means that there are no localized nodes near to download the file from. In this case TopBT attempts 


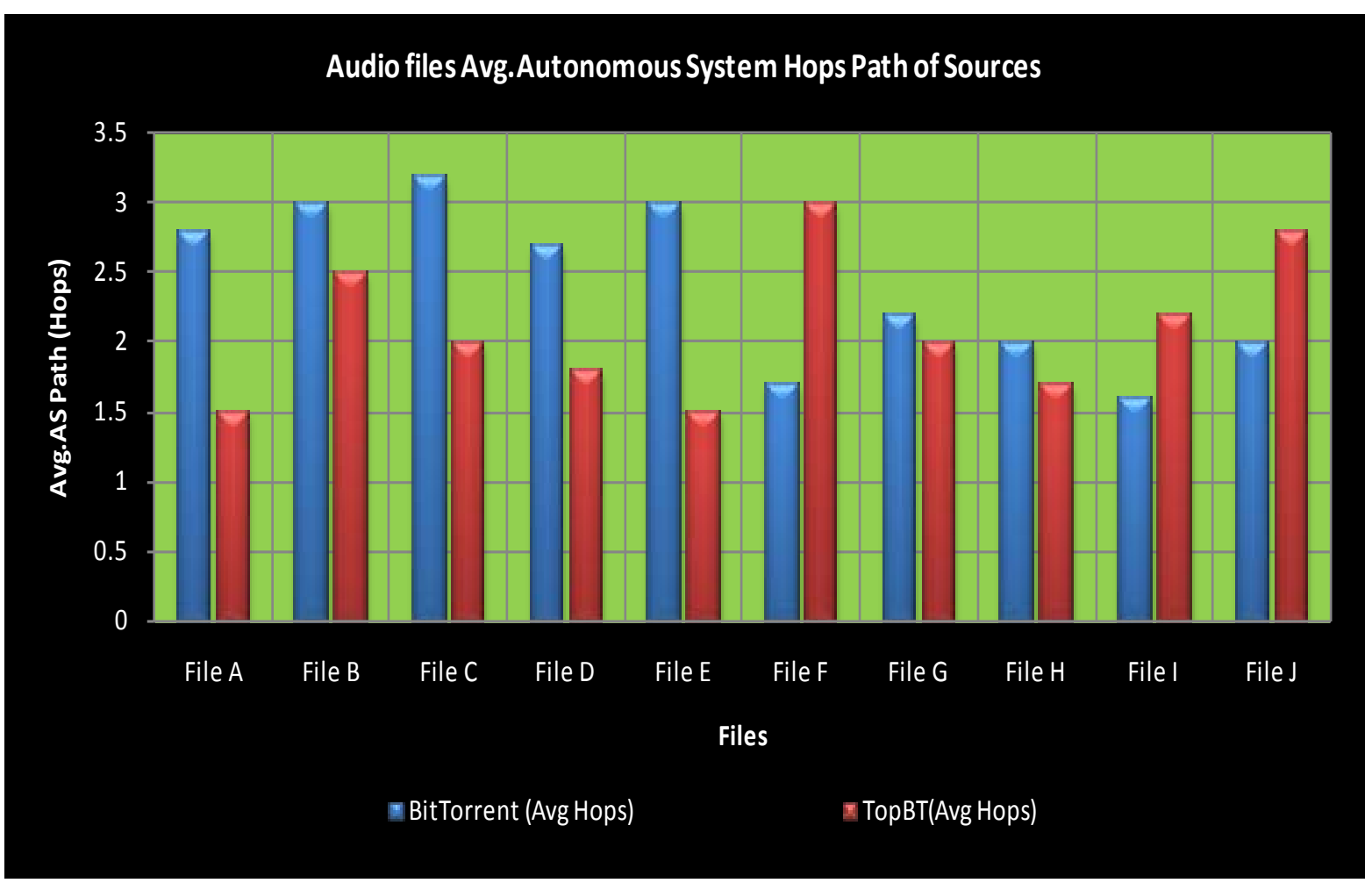

Figure 1. Source autonomous system hops path for audio files.

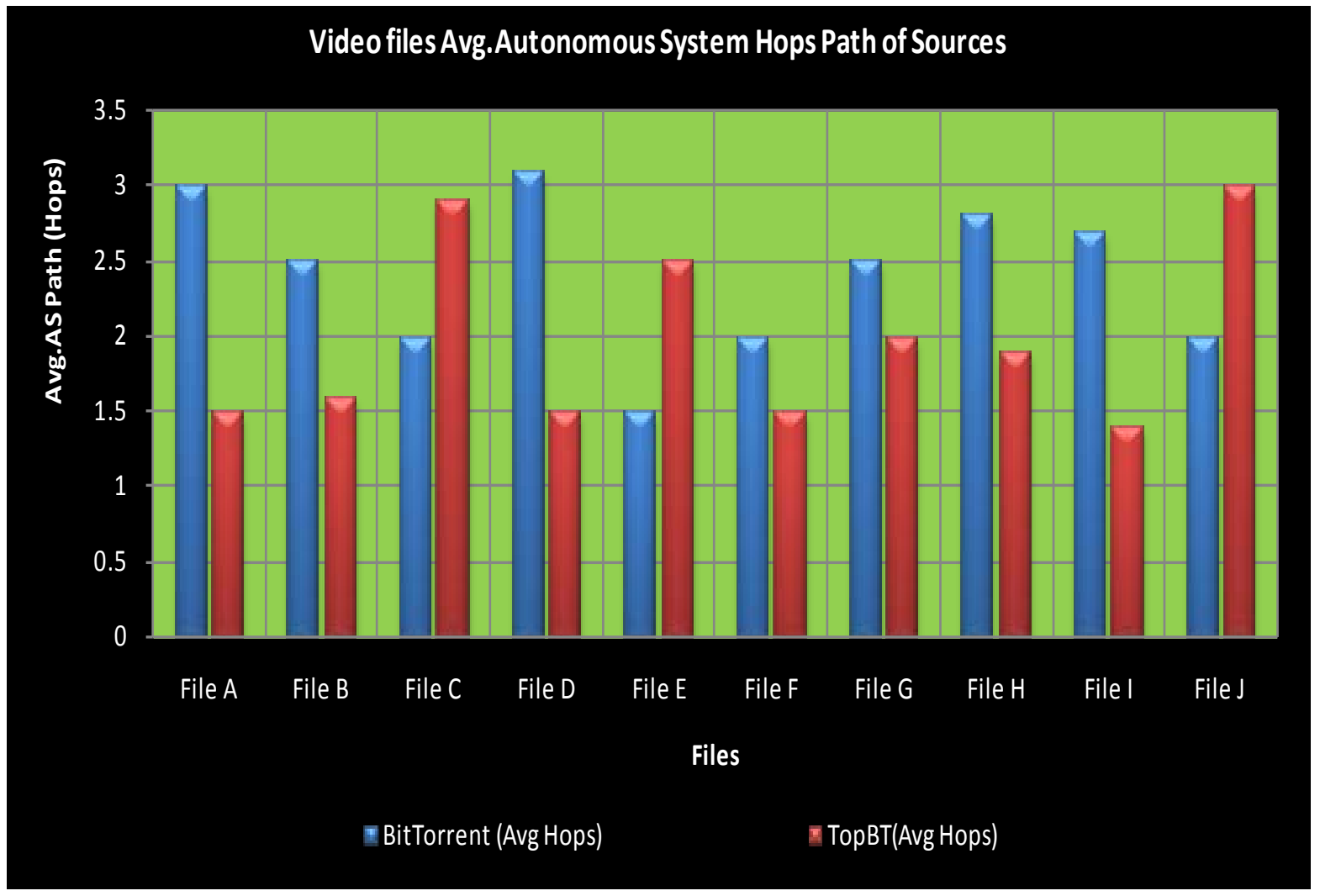

Figure 2. Source autonomous system hops path for Video files. 


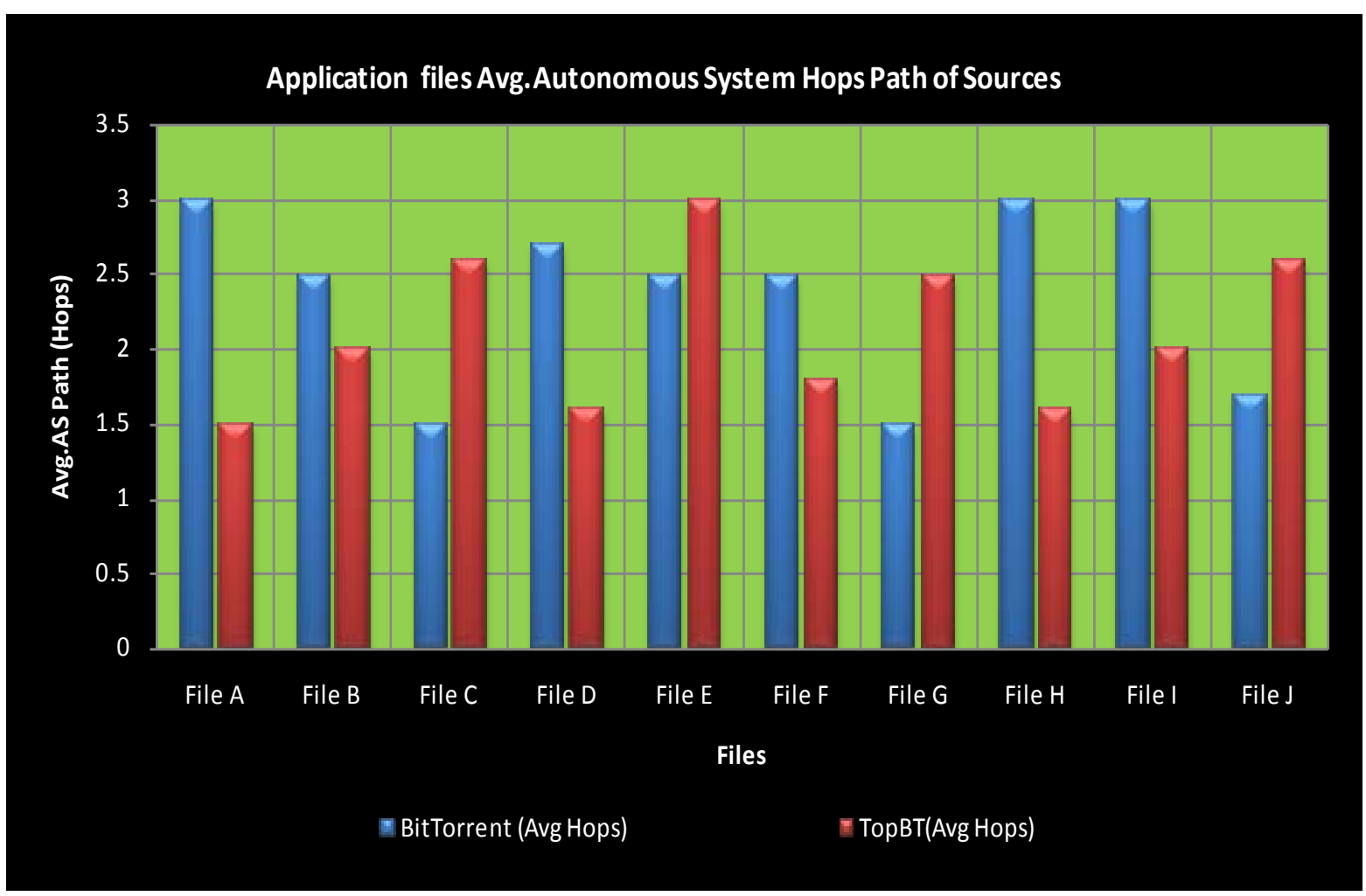

Figure 3. Source autonomous system hops path for application files.

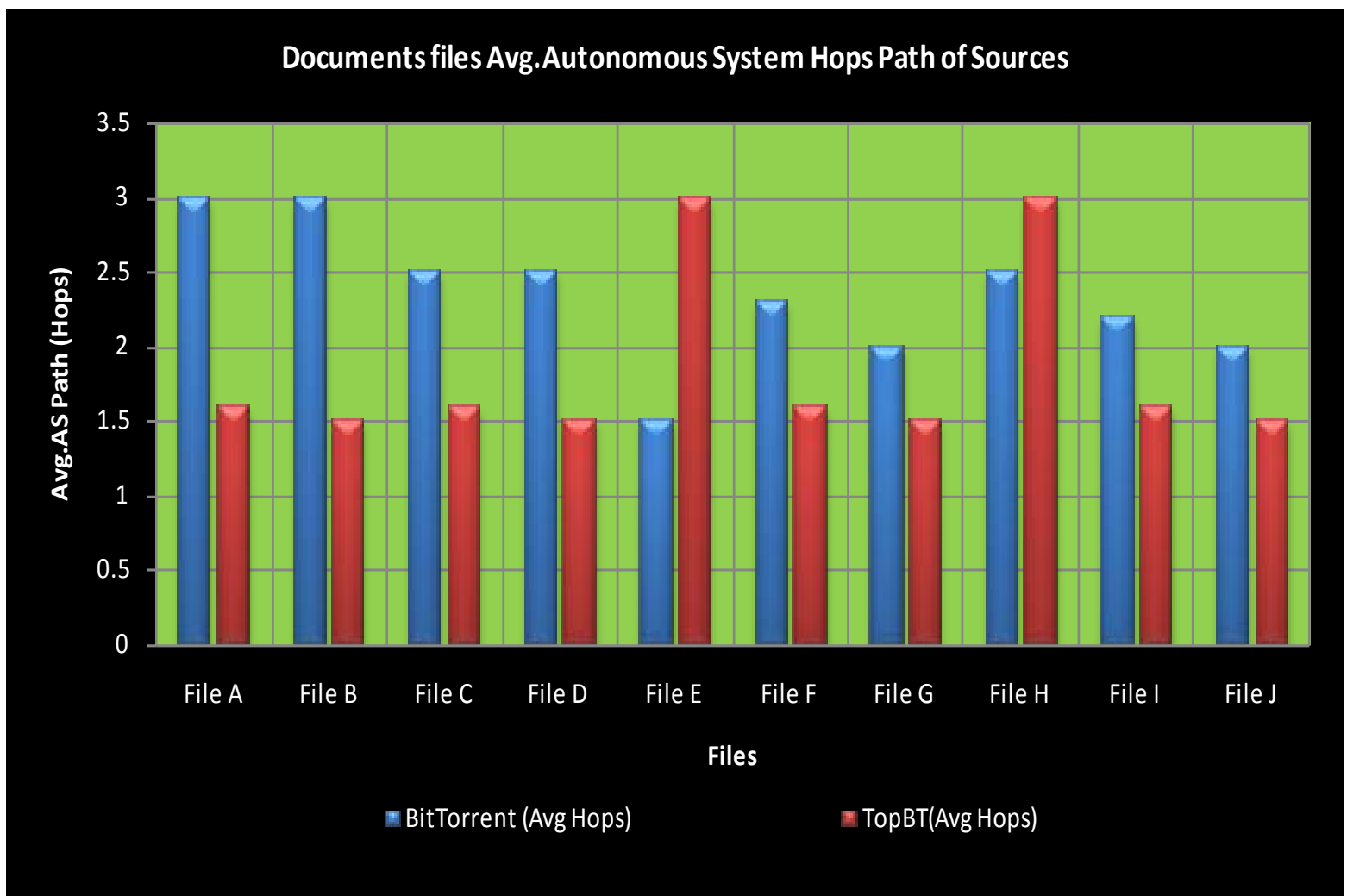

Figure 4. Source autonomous system hops path for documents files. 


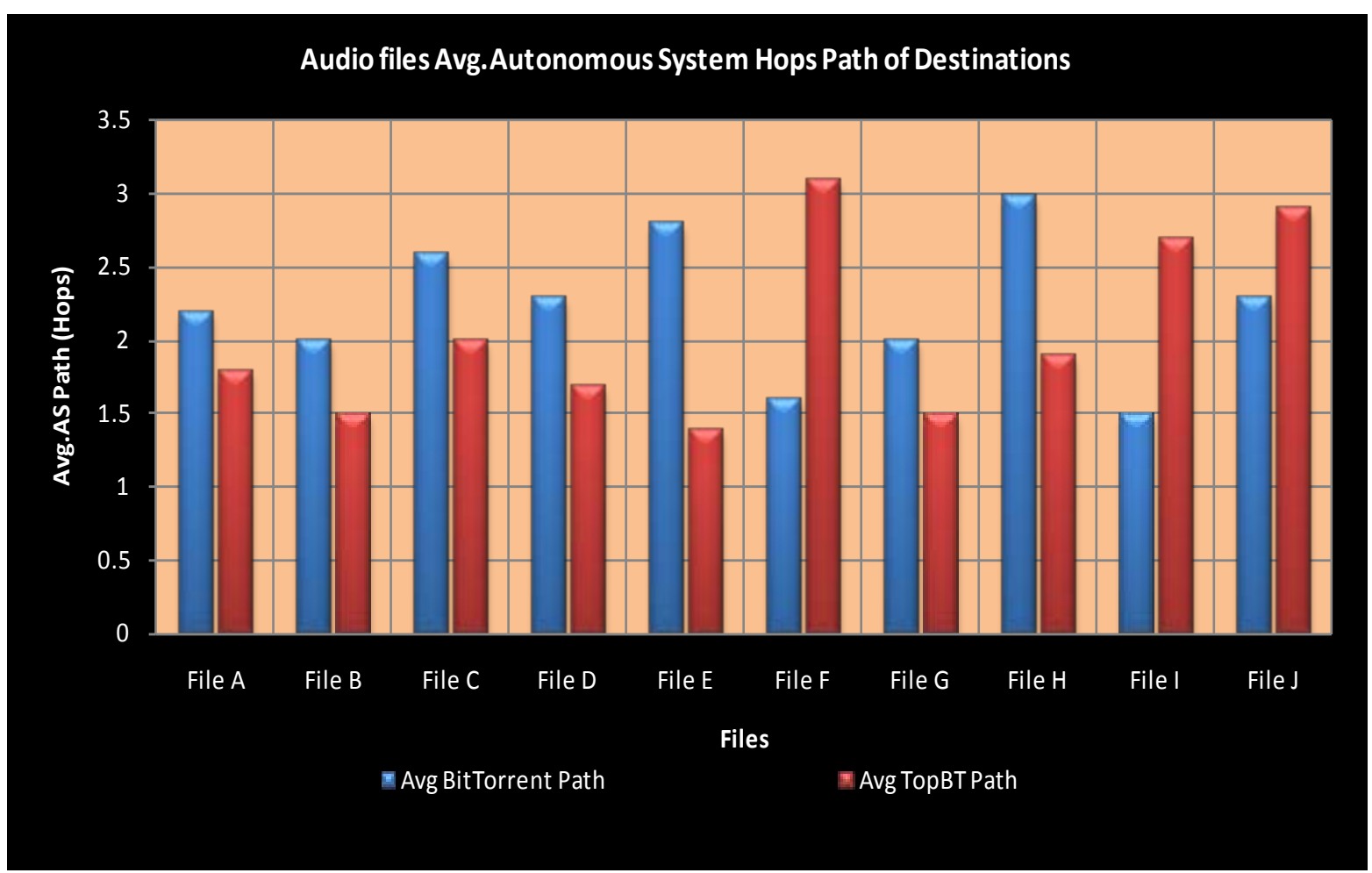

Figure 5. Destination autonomous system hops path for audio files.

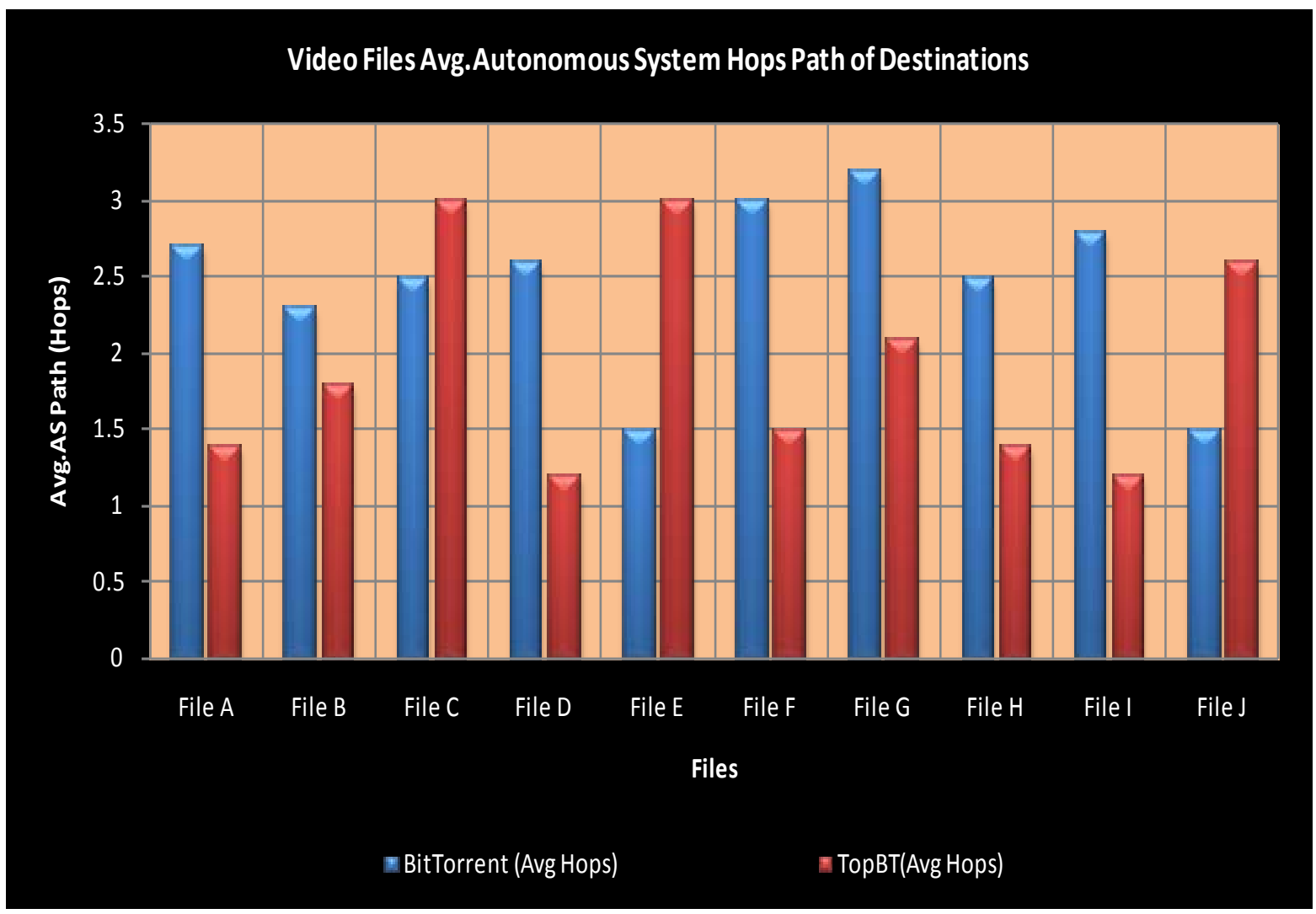

Figure 6. Destination autonomous system hops path for video files. 


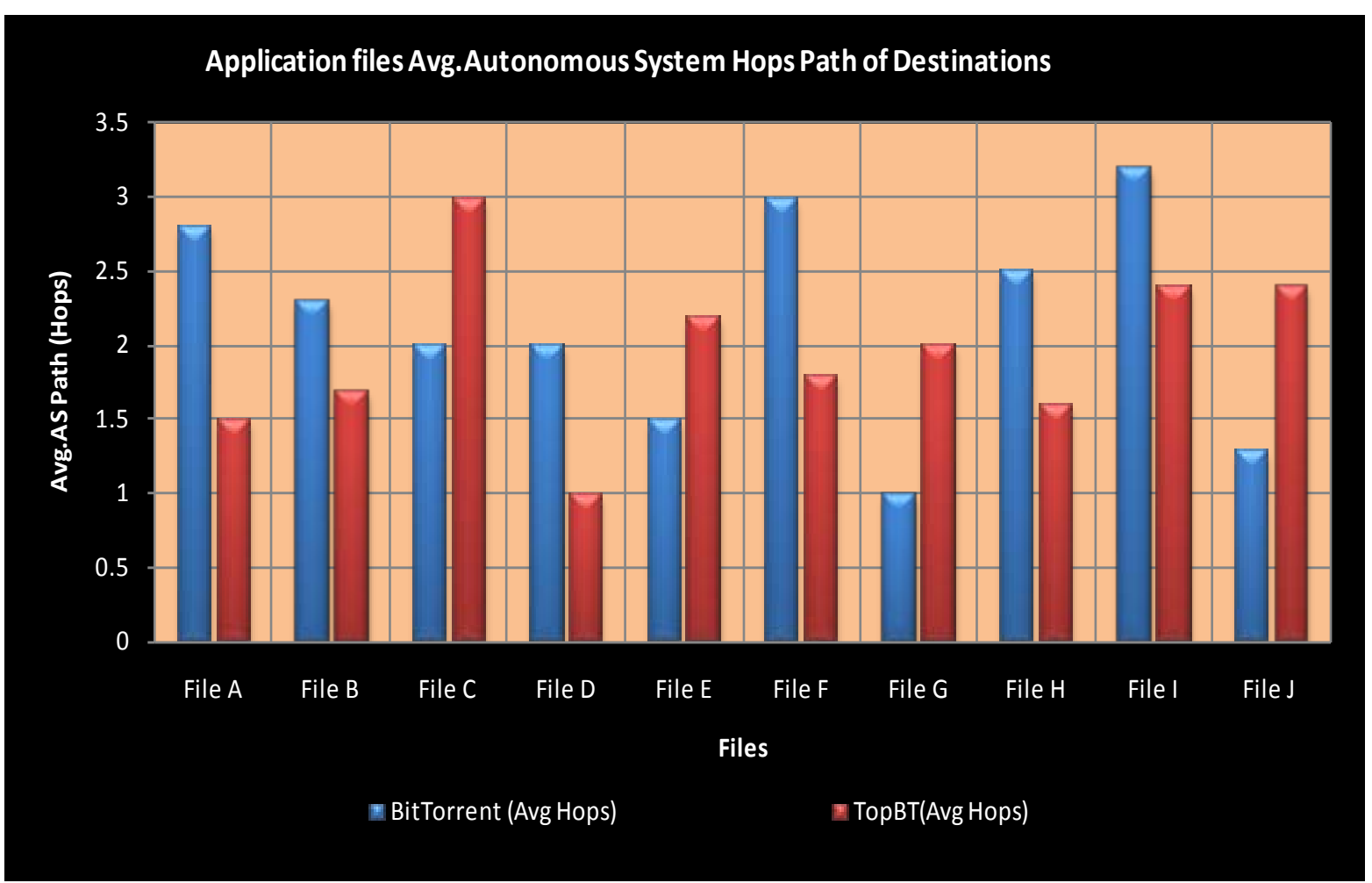

Figure 7. Destination autonomous system hops path for application files.

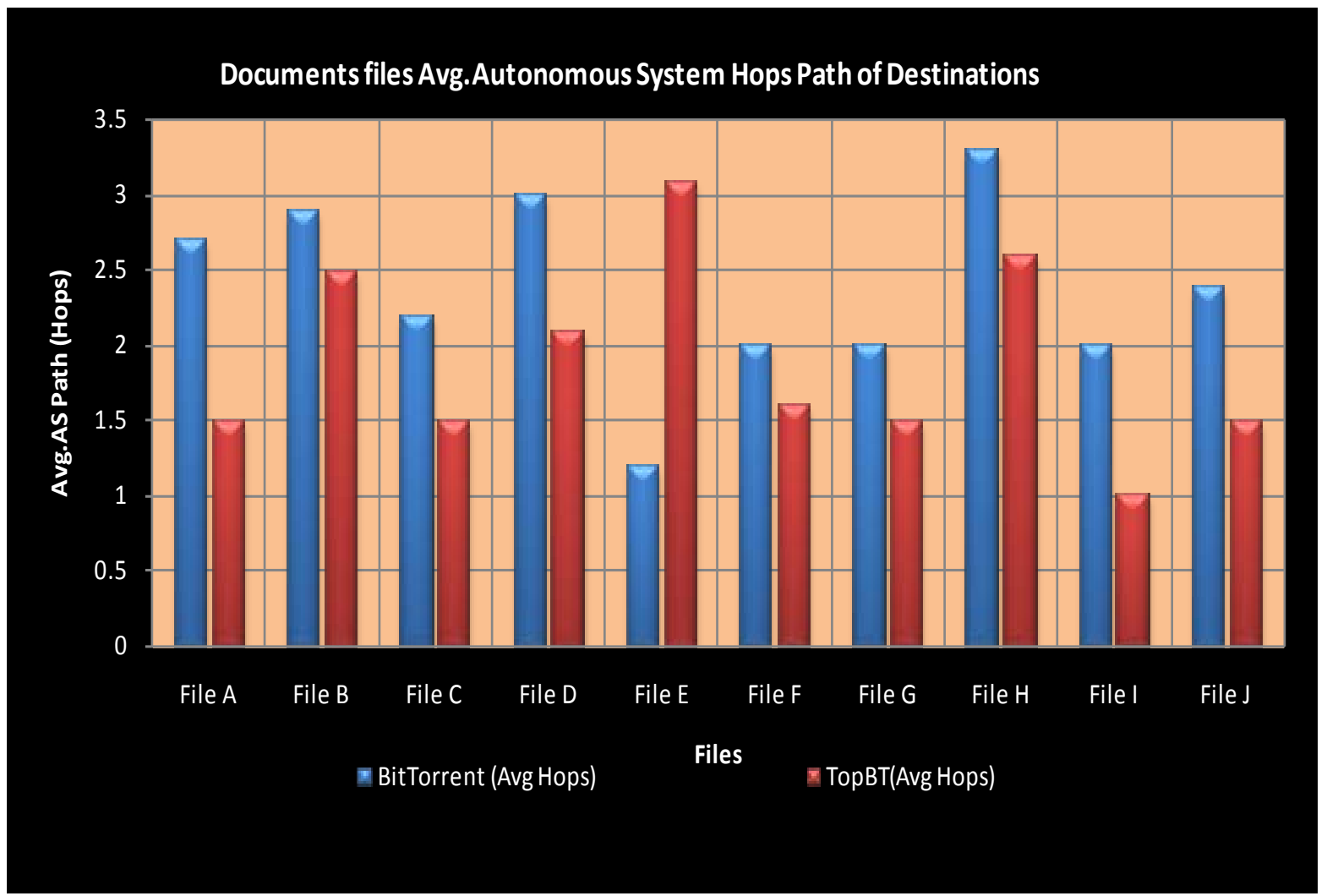

Figure 8. Destination autonomous system hops path for documents files. 
to download the files from faster nodes with highest upload bandwidth and hence this fact has increased the path for such scenarios. We can observe that the download time has reduced in many scenarios for TopBT, this shows that locality can also help in improving the Quality of Experience. Unfortunately, this is not the case for all files. TopBT attempts to reduce paths' length first which can affect the download time by downloading the file from closer procrastinate nodes.

\section{Conclusions \& Future Work}

In this work, we conducted a measurement study to investigate the advantages and drawbacks of implementing locality awareness algorithm in P2P networks and examined the locality awareness algorithm in BitTorrent and TopBT. We have compared the performance of TopBT with BitTorrent and utilized Wireshark tool to collect information from P2P network. In addition, an AS graph has been constructed to implement a shortest path algorithm to measure AS hops count between nodes in which collected peers from BitTorrent have been used as input to measure their destinations.

In future work, we can use other P2P applications and compare their results with each other and also investigate this measurement study in different locations and diverse ISP internet connections.

P2P model will remain dominating in coming years as we believe that research and development will continue to adapt P2P overlays which are more suitable for current internet infrastructure. P2P systems evolution will provide insights into the development of other large-scale distributed systems.

\section{References}

[1] Quang, H.V., Minhai, L. and Beng, C.O. (2010) Peer to Peer Computing Principles and Applications. New York City. http://dx.doi.org/10.1007/978-3-642-03514-2

[2] Masoud, M.Z., Hei, X.J. and Cheng, W.Q. (2012) Constructing a Locality-Aware ISP-Friendly Peer-to-Peer Live Streaming Architecture. Proceeding of International Conference on Information Science and Technology (ICIST), Hubei, 23-25 March 2012, 368-376. http://dx.doi.org/10.1109/ICIST.2012.6221670

[3] Alhaisoni, M., Liotta, A. and Ghanbari, M. (2009) Performance Analysis and Evaluation of P2PTV Streaming Behavior. International Symposium on Computers and Communications (ISCC), Sousse, 89-94. http://dx.doi.org/10.1109/ISCC.2009.5202402

[4] Zhang, D.Y., Zheng, C., Zhang, H.L. and Yu, H.L. (2010) Identification and Analysis of Skype Peer-to-Peer Traffic. International Conference on Internet and Web Applications and Services (ICIW), Barcelona, 200-206. http://dx.doi.org/10.1109/ICIW.2010.36

[5] Aggarwal, V., Akonjang, O. and Feldmann, A. (2008) Improving User and ISP Experience through ISP-Aided P2P Locality. Proceedings of INFOCOM Workshops, Phoenix, 1-6. http://dx.doi.org/10.1109/INFOCOM.2008.4544640

[6] Ren, S., Tan, E., Luo, T., Guo, L., Chen, S. and Zhang, X. (2010) TopBT: A Topology-Aware and Infrastructure-Independent BitTorrent Client. Proceedings of INFOCOM, San Diego, 1-9. http://dx.doi.org/10.1109/INFCOM.2010.5461969

[7] Xia, R.L. and Muppala, J.K. (2010) A Survey of BitTorrent Performance. International Journal of Communications Surveys \& Tutorials, 12, 140-158. http://dx.doi.org/10.1109/SURV.2010.021110.00036

[8] Fras, M., Klampfer, S. and Cucej, Z. (2008) Impact of P2P Traffic on IP Communication Network Performances. Proceeding of International Conference on Systems, Signals and Image Processing, Bratislava, 205-208. http://dx.doi.org/10.1109/IWSSIP.2008.4604403

[9] Blond, S.L., Legout, A. and Dabbous, W. (2004) Pushing BitTorrent Locality to the Limit. International Journal of Computer and Telecommunications Networking, 55, 541-557. http://dx.doi.org/10.1016/j.comnet.2010.09.014

[10] Coffins, D.R. and Bustamante, F.E. (2008) Taming the Torrent: A Practical Approach to Reducing Cross-ISP Traffic in Peer-to-Peer Systems. Proceeding of ACM SIGCOMM Conference on Data Communication, 363-374. http://dx.doi.org/10.1145/1402958.1403000

[11] Lewis, P.R., et al. (2011) A Survey of Self-Awareness and Its Application in Computing Systems. Proceeding of International Conference on Self-Adaptive and Self-Organizing Systems Workshops (ICSASOW), Ann Arbor, 102-107. http://dx.doi.org/10.1109/SASOW.2011.25

[12] Agarwal, V., Feldmann, A. and Scheideler, C. (2007) Can ISPs and P2P Users Cooperate for Improved Performance. ACM SIGCOMM Computer Communication Review Journal, 37, 29-40.

[13] Chomsiri, T. (2008) Sniffing Packets on LAN without ARP Spoofing. Proceeding of International Conference on Convergence and Hybrid Information Technology (ICCIT), 2, 472-477. http://dx.doi.org/10.1109/ICCIT.2008.318 
[14] Wang, S.Q., Xu, D.S. and Yan, S.L. (2010) Analysis and Application of Wireshark in TCP/IP Protocol Teaching. Proceeding of International Conference on E-Health Networking Digital Ecosystems and Technologies (EDT), 2, $269-272$. http://dx.doi.org/10.1109/EDT.2010.5496372

[15] Liu, B., Cui, Y., Lu, Y.S. and Xue, Y. (2009) Locality-Awareness in BitTorrent-Like P2P Applications. IEEE Transactions on Multimedia-Special Section on Communities and Media Computing Journal, 11, 361-371. http://dx.doi.org/10.1109/TMM.2009.2012911

[16] Aho, A.V., Kernighan, B.W. and Weinberger, P.J. (1988) The AWK Programming Language. New York.

[17] Cymru. http://www.team-cymru.org/Services/ip-to-asn.html 
Scientific Research Publishing (SCIRP) is one of the largest Open Access journal publishers. It is currently publishing more than 200 open access, online, peer-reviewed journals covering a wide range of academic disciplines. SCIRP serves the worldwide academic communities and contributes to the progress and application of science with its publication.

Other selected journals from SCIRP are listed as below. Submit your manuscript to us via either submit@scirp.org or Online Submission Portal.
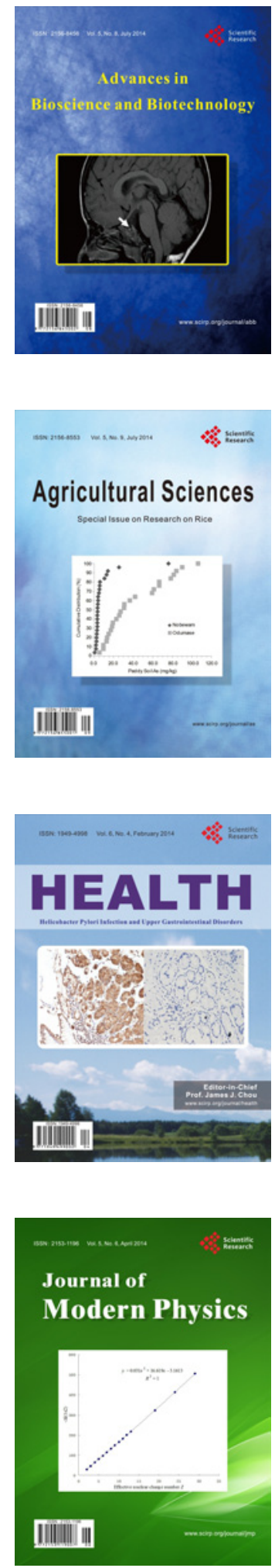
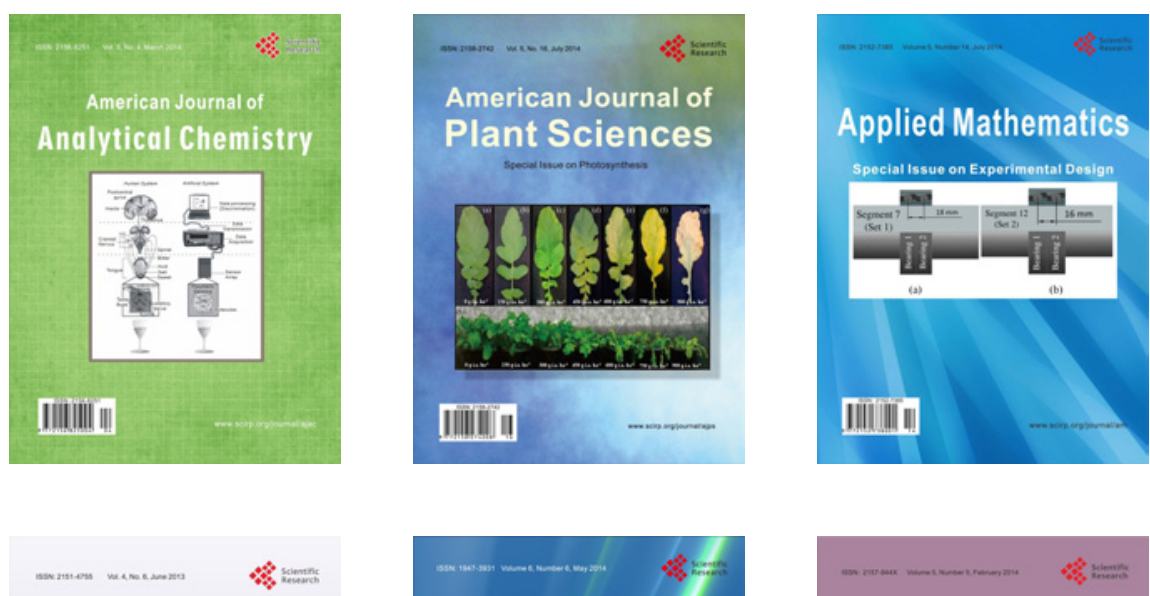

Creative Education
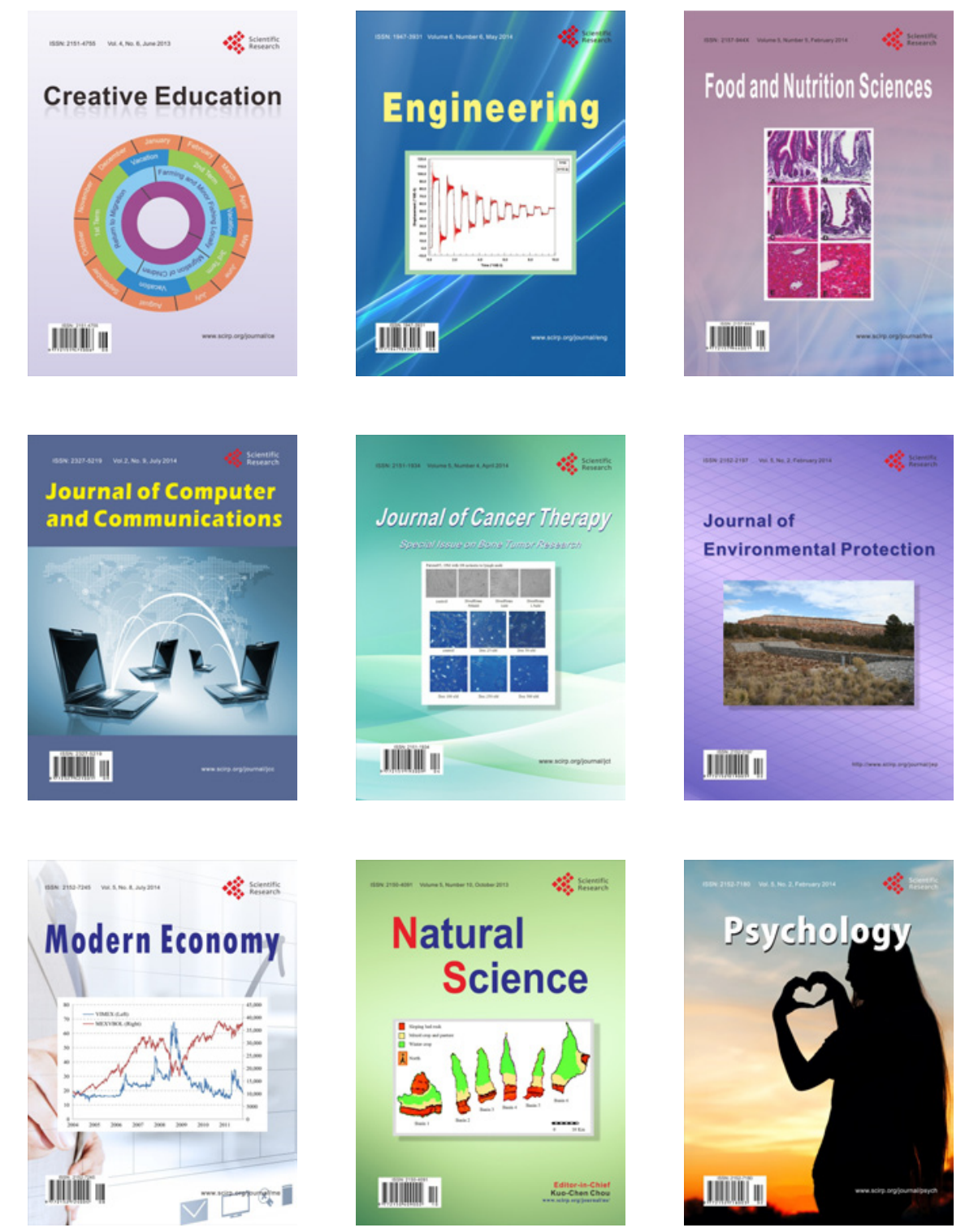